\title{
Design of Two Lightweight, High-Bandwidth Torque-Controlled Ankle Exoskeletons
}

\author{
Kirby Ann Witte ${ }^{1}$, Juanjuan Zhang ${ }^{1,2}$, Rachel W. Jackson ${ }^{1}$, Steven H. Collins ${ }^{1,3, *}$
}

\begin{abstract}
Lower-limb exoskeletons capable of comfortably applying high torques at high bandwidth can be used to probe the human neuromuscular system and assist gait. We designed and built two tethered ankle exoskeletons with strong lightweight frames, comfortable three-point contact with the leg, and series elastic elements for improved torque control. Both devices have low mass $(<0.88 \mathbf{~ k g})$, are modular, structurally compliant in selected directions, and instrumented to measure joint angle and torque. The exoskeletons are actuated by an off-board motor, and torque is controlled using a combination of proportional feedback and damping injection with iterative learning during walking tests. We tested closed-loop torque control by commanding $50 \mathrm{~N} \cdot \mathrm{m}$ and $20 \mathrm{~N} \cdot \mathrm{m}$ linear chirps in desired torque while the exoskeletons were worn by human users, and measured bandwidths greater than $16 \mathrm{~Hz}$ and $21 \mathrm{~Hz}$, respectively. During walking trials, we demonstrated $120 \mathrm{~N} \cdot \mathrm{m}$ peak torque and $\mathbf{2 . 0} \mathrm{N} \cdot \mathrm{m}$ RMS torque tracking error. These performance measures compare favorably with existing devices and with human ankle musculature, and show that these exoskeletons can be used to rapidly explore a wide range of control techniques and robotic assistance paradigms as elements of versatile, high-performance testbeds. Our results also provide insights into desirable properties of lower-limb exoskeleton hardware, which we expect to inform future designs.
\end{abstract}

Index Terms-Rehabilitation Robotics, Human-Robot Interaction, Ankle Exoskeleton

\section{INTRODUCTION}

Lower-limb exoskeletons have the potential to aid in rehabilitation [1], assist walking for those with gait impairments [2], reduce the metabolic cost of normal [3] and load-bearing walking [4,5], improve stability [6] and probe interesting questions about human locomotion [4]. The challenges of designing effective lower-limb exoskeletons may be simplified by focusing on a single joint. During normal walking, the ankle produces a larger peak torque and performs more positive work than either the knee or the hip [7]. The ankle joint may therefore prove an effective location for application of assistance. [8, 9]

Many exoskeletons have been developed employing different approaches to mechanical design, actuation, and control $[3,4,5,10]$. Though the most effective mechanical method to assist the ankle remains unclear, the process of designing and testing our devices has produced several guiding principles for exoskeleton design.

This material is based upon work supported by the National Science Foundation under Grant No. IIS-1355716.

${ }^{1}$ Dept. Mechanical Engineering, Carnegie Mellon University, USA.

${ }^{2}$ School of Electrical and Electronic Engineering, Nanyang Technological University, Singapore.

${ }^{3}$ Robotics Institute, Carnegie Mellon University, USA.

${ }^{*}$ Corresponding author: S. H. Collins. 5000 Forbes Ave. Pittsburgh, Pennsylvania 15213, USA. Email:stevecollins@cmu.edu
Delivering positive work with an exoskeleton by supplying ankle plantarflexor torques can reduce the metabolic energy cost of normal [3] and load bearing walking [11]. Increasing the amount of net work supplied by the device results in a downward trend in metabolic energy cost [12]. The ankle joint experiences a wide range of velocities during normal walking, with plantarflexion occurring rapidly. The ability to apply large torques and do net work therefore enriches the space of potential assistance techniques, and allows the device to keep up with natural movements of the user. Independent of maximum torque, the system's responsiveness to changes in desired torque is important. For example, the timing of torque application in the gait cycle strongly affects metabolic energy consumption [13].

Effective design of exoskeletons requires an understanding of human-device interaction. The device must be able to transfer loads comfortably, quickly, effectively, and safely. Shear forces cause discomfort when interfacing with skin. Applying forces normal to the human over large surface areas allows for greater magnitudes of applied force while maintaining comfort. Applying forces far from the ankle joint, thereby increasing the lever arm, reduces the magnitude of applied force necessary for a desired externally applied ankle torque. Series elasticity improves torque control and decouples the human from the inertia of the motor and gearbox [14]. The stiffness of the spring also determines the nominal behavior of the device, or the torque profile produced when the motor position is held constant while ankle angle changes. The optimal stiffness is not known a priori as it may vary across subjects and applications, and experiments should be performed to determine the appropriate spring stiffness. We must consider not only comfort, but how the system changes with human interaction. While an exoskeleton may have high torque and bandwidth capabilities on a test stand, results may change when a human is included in the system.

Many ankle exoskeletons are designed to reduce metabolic energy cost. Placing an ankle exoskeleton on the leg, however, automatically incurs a metabolic energy penalty because it adds distal mass [15, 16]. Reducing total device mass helps decrease this penalty. Ankle exoskeletons also interfere with natural motion and, although this problem can be partially addressed by good control, some interference is unavoidable due to the physical structure of the device. Maintaining compliance in uncontrolled directions, such as inversion and eversion, allows for less inhibited motion. Reducing the overall device envelope, especially the width, decreases additional metabolic energy costs associated 
with increased step width [17]. Users may vary greatly in anthropometry, such as body mass and leg length. Rather than designing a new device for each user [4], which is time-consuming and expensive, incorporating adjustability or modularity allows a single exoskeleton to be used on multiple subjects.

Human locomotion is a versatile and complex behavior that remains poorly understood, and designing devices to interact usefully with humans during walking is a difficult task. Building adjustable devices to supply a wide range of torques using numerous control schemes provides freedom to rapidly and inexpensively measure the human response to different strategies. Results from human experiments can provide insights into useful capabilities for future designs. Our goal was to develop a system that demonstrates effective solutions to challenges inherent in the design of ankle exoskeletons.

\section{MEthods}

We designed, built and tested two ankle exoskeletons to be used as end-effectors in a tethered emulator system (Fig. 1A). The prototypes, Alpha and Beta, demonstrate two approaches to exoskeleton design, including fabrication of strong, lightweight components, implementation of series elasticity for improved torque control, and comfortable interfacing that reduces restriction of natural movement.

The Alpha exoskeleton was designed to provide compliance in selected directions. The Beta exoskeleton was designed to reduce overall envelope. Benchtop tests quantified system-wide closed-loop torque bandwidth, and walking trials quantified torque tracking error while verifying that large torques could be comfortably applied.

\section{A. Mechanical Design}

The ankle exoskeleton end-effectors were actuated by a powerful off-board motor and real-time controller, with mechanical power transmitted through a flexible Bowden cable tether. The motor, controller and tether elements of this system are described in detail in [18].

Both ankle exoskeletons interface with the foot under the heel, the shin below the knee, and the ground beneath the toe. The exoskeleton frames include rotational joints on either side of the ankle, with axes of rotation approximately collinear with that of the human joint (Fig. 1 B,C).

Each frame can be separated into foot and shank sections (Fig. 1 B,C). The foot section has a lever arm posterior to the ankle that wraps around the heel. The Bowden cable pulls up on this lever while the Bowden cable conduit presses down on the shank section. This results in an upward force beneath the user's heel, a normal force on the top of the shin, and a downward force on the ground, generating a plantarflexion torque (Fig. 2). The toe and shin attachment points are located far from the ankle joint, maximizing their leverage about the ankle and minimizing forces applied to the user for a given plantarflexion torque. Forces are comfortably transmitted to the shin via a padded strap, which is situated above the calf muscle to prevent the device from
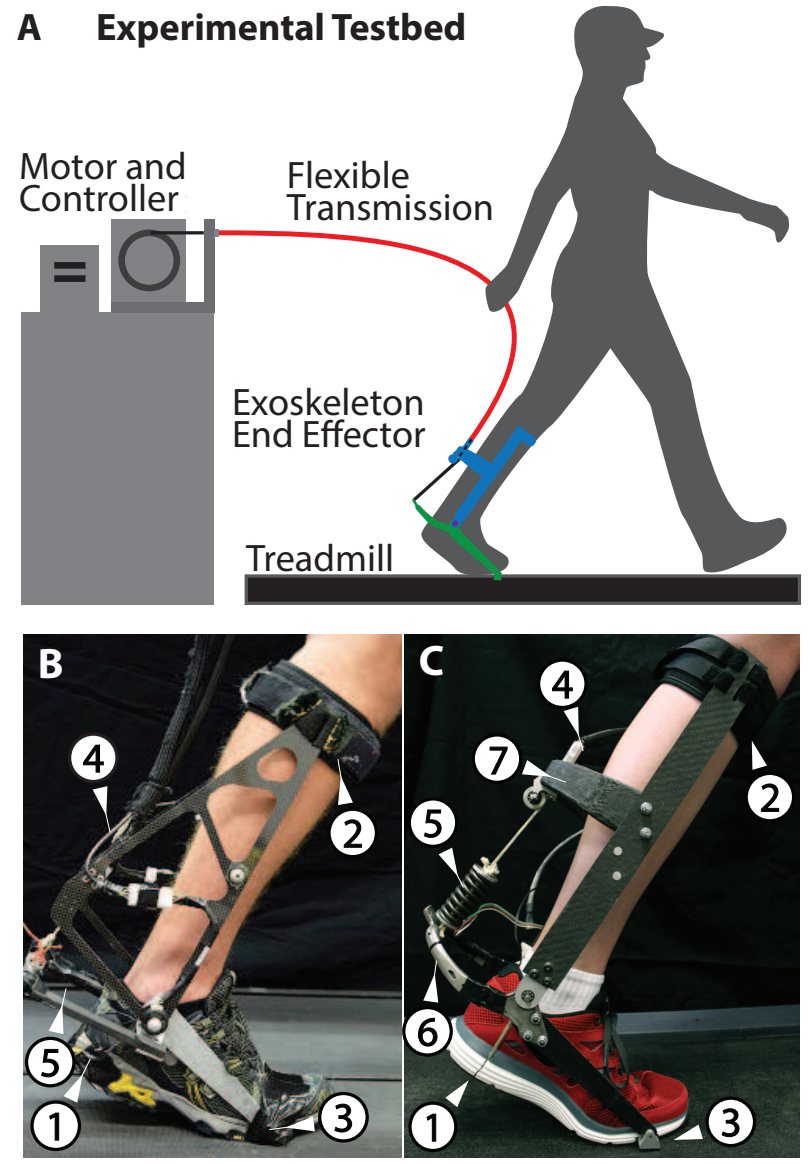

Fig. 1. Emulator system and exoskeleton end-effectors. A The testbed comprised a powerful off-board motor and controller, a flexible transmission, and an ankle exoskeleton end-effector worn on the person's leg. B The Alpha design. Each exoskeleton contacted (1) the heel using a string, (2) the shin using a strap, and (3) the ground using a hinged plate embedded in the shoe. The Bowden cable conduit attached to (4) the shank frame, while the Bowden cable rope terminated at (5) the series spring. C The Beta design. In addition to (1-5), this prototype has (6) a titanium ankle lever wrapping behind the heel and (7) a hollow carbon fiber Bowden cable support.

slipping down. Forces are transmitted to the user's heel via a lightweight synthetic rope placed in a groove in the sole of a running shoe.

The exoskeletons were designed to provide greater peak torque, peak velocity and range of motion than observed at the ankle during unaided fast walking. The Alpha and Beta devices can withstand peak plantarflexion torques of $120 \mathrm{~N} \cdot \mathrm{m}$ and $150 \mathrm{~N} \cdot \mathrm{m}$ respectively. The expected peak plantarflexion velocities, limited by motor speed, of the Alpha and Beta devices are 300 and $303 \mathrm{deg} \cdot \mathrm{s}^{-1}$, respectively. Both devices have a range of motion of $30^{\circ}$ plantarflexion to $20^{\circ}$ dorsiflexion, with $0^{\circ}$ corresponding to a natural standing posture.

Both exoskeletons are modular to accommodate a range of subject sizes. Toe struts, calf struts, and heel strings can be exchanged to fit different foot and shank sizes. Current hardware fits users with shank lengths ranging from 0.42 to $0.50 \mathrm{~m}$ and shoe sizes ranging from a women's size 7 to a men's size 12 (US). Slots in the calf struts allow an additional $0.04 \mathrm{~m}$ of continuous adjustability in the Beta device.

Series elasticity was provided by a pair of leaf springs in 

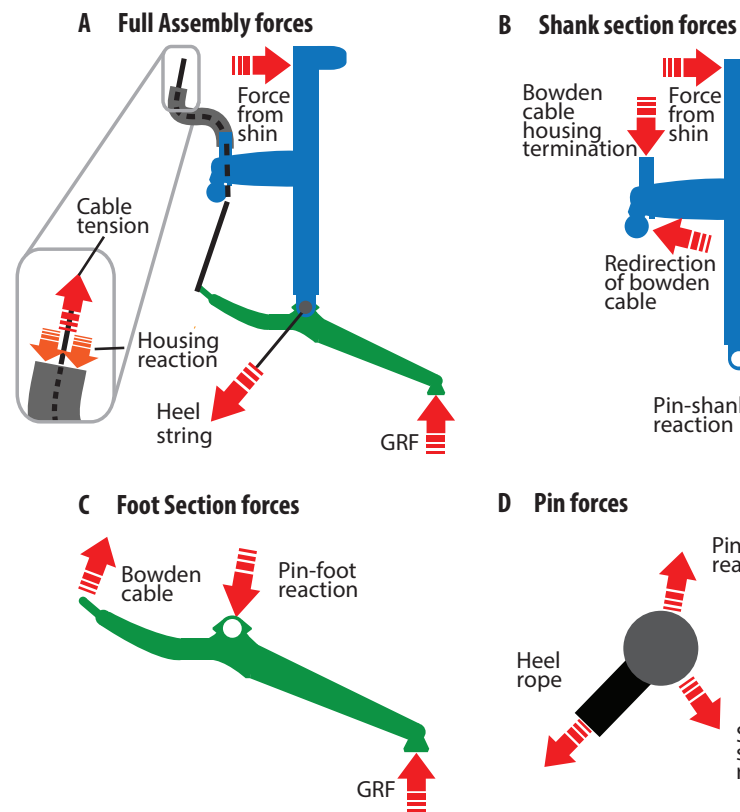

D Pinforces

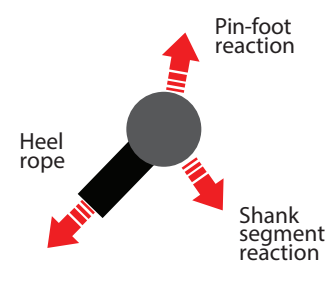

Fig. 2. Free body diagrams of the exoskeleton structure. A The complete exoskeleton experiences external loads at the three attachment points, which together create an ankle plantarflexion torque. Forces in the Bowden cable conduit and inner rope (inset) are equal and opposite, producing no net external load on the leg. Free body diagrams depicting loading of $\mathbf{B}$ the shank segment, $\mathbf{C}$ the foot segment, and $\mathbf{D}$ the shaft and heel rope.

the Alpha design. The custom leaf springs were fiberglass (GC-67-UB, Gordon Composites, Montrose, CO, USA), which has a mass per unit strain-energy storage, $\rho E \sigma_{y}^{-2}$, one eighth that of spring steel [18], but mass savings are limited by additional hardware needed for robust attachment. The leaf springs also functioned as the ankle lever in the Alpha exoskeleton, thereby reducing the number of components required. A stock coil spring (DWC-225M-13, Diamond Wire Spring Co., Pittsburgh, PA, USA) was used in the Beta design. The lever arm and joint assembly of the Alpha device was lighter by $0.059 \mathrm{~kg}$ compared to the Beta design, but this comparison is confounded by factors such as different maximum expected loads and spring stiffnesses.

Spring type strongly affected overall exoskeleton envelope. The structure of the Alpha device extends substantially into space medial and posterior to the ankle joint (Fig. 3). This large envelope increased user step width [12], potentially increasing metabolic energy consumption during walking [19], and caused occasional collisions with the contralateral limb. The average maximal ankle external rotation during walking for healthy subjects is approximately $18^{\circ}$ [20], and the average step width is only $0.1 \mathrm{~m}$ [21]. For this reason, the Beta exoskeleton was designed to reduce medial and lateral protrusions to prevent collisions and excessive widening of step width during bilateral use. The maximum protrusion length measured from the center of the human ankle joint is $24 \%$ smaller than that of the Alpha design.

The Alpha design's plate-like components were easily machined, while more complex Beta components were suited to additive manufacturing and lost-wax carbon fiber molding. The Beta Exoskeleton originally featured a leaf spring extending from the ankle lever. Due to this configuration,

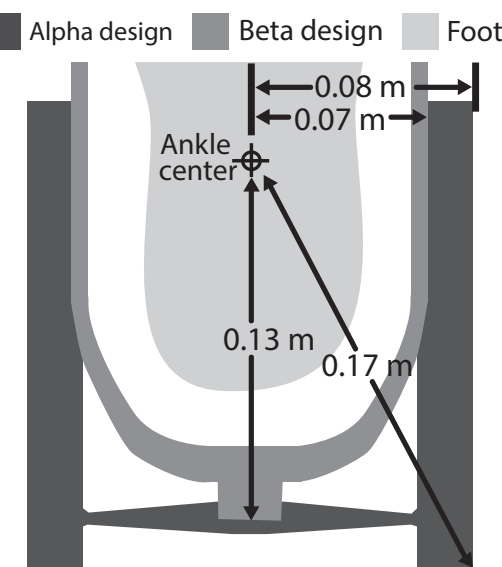

Fig. 3. Comparison of envelopes of the two devices, depicted from above The Beta device was slimmer in terms of medial-lateral protrusion and maximum protrusion from the joint center.

the lever experienced large bending and torsion loads, well addressed by I-beam and tubular structures. The ankle lever also required small, precise features for connection to the ankle shaft and toe hardware. Additive manufacturing using electron sintering of titanium allowed these disparate design requirements to be addressed by a single component. The titanium component weighed $0.098 \mathrm{~kg}$ less than an equivalent structure from an earlier prototype comprised of a carbon fiber ankle lever, two aluminum joint components, a fiberglass leaf spring, and connective hardware. The Beta Bowden cable termination support is subjected to similar loading as the ankle lever, but has less complex connection geometry, making a hollow carbon fiber structure appropriate. This part was manufactured using a lost wax molding method. A wax form with a threaded aluminum insert was cast using a fused deposition ABS shell-mold. A composite layup was performed on the wax form using braided carbon fiber sleeves. The wax was melted out by submerging the component in warm water. In an earlier prototype, we performed the carbon fiber layup on a hollow plastic mold, reinforced to withstand the vacuum bagging process. This permanent plastic mold added approximately $0.048 \mathrm{~kg}$ to the component.

Both exoskeleton designs provide some structural compliance. Thin plate-like shank struts act as flexures, allowing the calf strap to fit snugly around a wide range of calf sizes and move medially and laterally. This flexural compliance, in concert with sliding of the calf strap on the struts, sliding of the rope beneath the heel, and compliance in the shoe, allows ankle rotation in both roll and yaw during walking. The Bowden cable support connecting the medial and lateral shank struts is located lower and further back from the leg in the Alpha design, allowing more deflection at the top of the struts. The Bowden cable support is located higher in the Beta design to allow space for the in-line coil spring, which reduces compliance near the calf strap and makes additional spacers necessary to appropriately fit smaller calves.

\section{B. Sensing and Control}

Both devices sense ankle angle with optical encoders (E4P and E5, respectively, US Digital Corp., Vancouver, WA, 


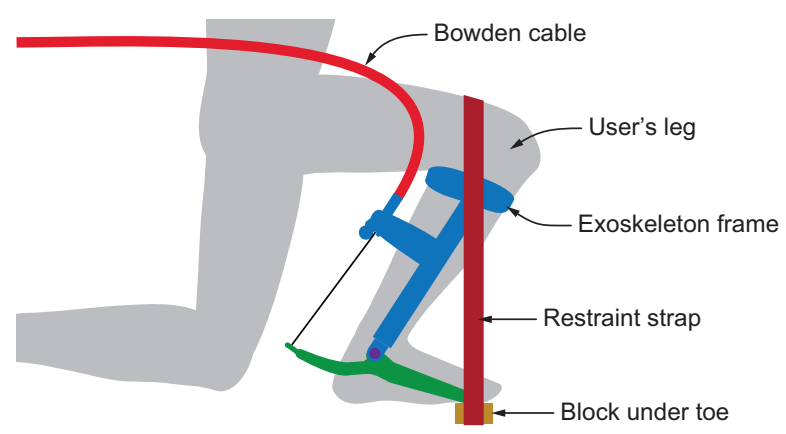

Fig. 4. Bandwidth test setup. The exoskeleton was worn by a human subject, whose leg was restrained using a strap that wrapped over the thigh and attached to a block beneath the toe segment.

USA) and foot contact with switches (7692K3, McMasterCarr, Cleveland, Ohio, USA) in the heel of the shoe. The Alpha exoskeleton uses a load cell (LC201, Omega Engineering Inc., Stamford, CT, USA) to measure Bowden cable tension. The Beta exoskeleton uses four strain gauges (MMF003129, Micro Measurements, Wendell, NC, USA) in a Wheatstonebridge on the ankle lever to measure torque directly. Bridge voltage was sampled at $5000 \mathrm{~Hz}$ and low-pass filtered at $200 \mathrm{~Hz}$ to reduce the effects of electromagnetic interference.

A combination of classical feedback control and iterative learning was used to control exoskeleton torque during walking. Proportional control with damping injection was used in closed-loop bandwidth tests. This approach is described in detail in [22].

For walking tests, desired torque was computed as a function of ankle angle and gait cycle phase. During stance, desired torque roughly matched the average torque-angle relationship of the ankle during normal walking (using a control method described in detail in [18]). During swing, a small amount of slack was maintained in the Bowden cable, resulting in no torque.

\section{Experimental Methods}

Torque sensors were calibrated by removing and securing the ankle lever upside down in a jig. Torque was incrementally increased by hanging weights of known mass from the Bowden cable. We computed root mean squared (RMS) error between applied and measured torque from the calibration set.

We performed closed-loop torque bandwidth tests on the ankle exoskeleton while worn by a user. This captured the effects of soft tissues and compliance in the shoe on torque control. The user's ankle was restrained by a strap that ran under the toe and over the knee (Fig. 4, Supplementary Video). Linear chirps in desired torque were applied with a maximum frequency of $30 \mathrm{~Hz}$ over a 30 second period, and measured torque was recorded. Bode frequency response plots were generated using the Fourier transform of desired and measured torque signals. Ten tests were performed at amplitudes of 20 and $50 \mathrm{~N} \cdot \mathrm{m}$, and results were averaged. Bandwidth was calculated as the lesser of the $-3 \mathrm{~dB}$ cutoff frequency and the $30^{\circ}$ phase margin crossover frequency.

Torque tracking performance was evaluated during walking trials with a single healthy subject $(1.85 \mathrm{~m}, 77 \mathrm{Kg}$,
TABLE I

MASS BREAKDOWN (KG)

\begin{tabular}{lcr} 
Assembly & Alpha & Beta \\
\hline Lever Arm, Spring and Joint & 0.256 & 0.315 \\
Struts and Bowden Cable Support & 0.258 & 0.312 \\
Toe Plates & 0.154 & 0.074 \\
Straps & 0.063 & 0.120 \\
Wiring and Sensors & 0.104 & 0.054 \\
Total & $\mathbf{0 . 8 3 5}$ & $\mathbf{0 . 8 7 5}$
\end{tabular}

35 yrs, male). Data was collected over 100 steady-state steps while walking on a treadmill at $1.25 \mathrm{~m} \cdot \mathrm{s}^{-1}$. RMS error was calculated over the entire trial and for an average step.

\section{RESULTS}

The total mass of the Alpha and Beta exoskeletons were 0.835 and $0.875 \mathrm{~kg}$, respectively (Table I). Torque measurement accuracy tests showed a RMS error of $0.751 \mathrm{~N} \cdot \mathrm{m}$ and $0.125 \mathrm{~N} \cdot \mathrm{m}$ for Alpha and Beta respectively (Fig. 5A).

The gain-limited closed-loop torque bandwidths of the Alpha device with $20 \mathrm{~N} \cdot \mathrm{m}$ and $50 \mathrm{~N} \cdot \mathrm{m}$ peak torques, were $21.1 \mathrm{~Hz}$ and $16.7 \mathrm{~Hz}$, respectively (Fig. 5B). The phaselimited bandwidths [23] for the Beta device, at a $30^{\circ}$ phase margin, with $20 \mathrm{~N} \cdot \mathrm{m}$ and $50 \mathrm{~N} \cdot \mathrm{m}$ peak torques were $24.2 \mathrm{~Hz}$ and $17.7 \mathrm{~Hz}$, respectively (Fig. 5B).

In walking trials with the Alpha device, the peak average measured torque was $80 \mathrm{~N} \cdot \mathrm{m}$. The maximum observed torque was $119 \mathrm{~N} \cdot \mathrm{m}$. The RMS error for the entire trial was $1.7 \pm 0.6 \mathrm{~N} \cdot \mathrm{m}$, or $2.1 \%$ of peak torque, and the RMS error of the average stride was $0.2 \mathrm{~N} \cdot \mathrm{m}$, or $0.3 \%$ of peak torque (Fig. 5C). For device Beta, the peak average measured torque was $87 \mathrm{~N} \cdot \mathrm{m}$. The maximum observed torque was $121 \mathrm{~N} \cdot \mathrm{m}$. The RMS error for the entire trial was $2.0 \pm 0.5 \mathrm{~N} \cdot \mathrm{m}$, or $2.4 \%$ of peak torque, and the RMS error of the average stride was $0.3 \mathrm{~N} \cdot \mathrm{m}$, or $0.4 \%$ of peak torque (Fig. $5 \mathrm{C}$ ).

\section{Discussion}

Our aim was to design comfortable, modular exoskeletons to provide high torque at high bandwidth with accurate torque tracking. Weighing less than $0.87 \mathrm{~kg}$, both exoskeletons compare favorably to a tethered pneumatic device used for probing the biomechanics of locomotion [4] and to an autonomous device for load carriage assistance [11]. The Alpha and Beta devices demonstrated a six-fold increase in bandwidth over a pneumatically actuated device that recently reduced metabolic energy consumption below that of normal walking [3]. Comparisons with other platforms are limited due to a lack of reported bandwidth values. In walking tests with users of varying shank lengths $(0.42 \mathrm{~m}$ to $0.50 \mathrm{~m})$, we observed peak torques of $120 \mathrm{~N} \cdot \mathrm{m}$, comparable to values from simliar devices $[3,4,10]$. These results demonstrate robust, accurate torque tracking and the ability to transfer large, dynamic loads comfortably to a variety of users.

Three-point contact with the user's leg implemented in both exoskeletons provided comfortable interfacing. Attachment point locations minimized the magnitude of forces applied to the body, while compliance in selected directions reduced interference with natural motions. Although differences in design led to more rigid struts in the Beta 

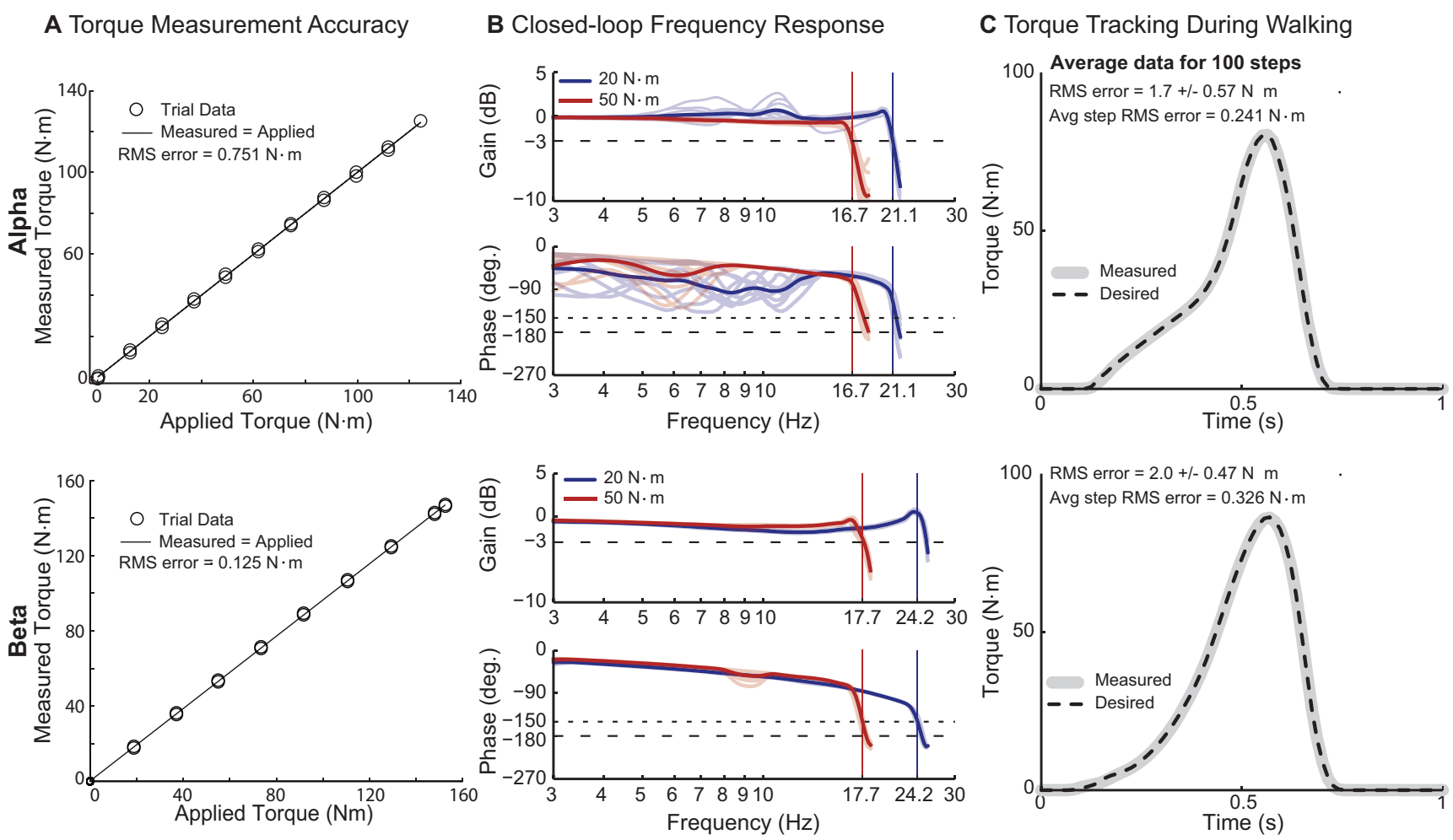

Fig. 5. Experimental results from tests of the Alpha (top) and Beta (bottom) prototypes. A Torque measurement calibration results. B Bode plots depicting frequency response of the system with peak desired torques of $20 \mathrm{~N} \cdot \mathrm{m}$ (blue) and $50 \mathrm{~N} \cdot \mathrm{m}$ (red). Bandwidth was gain-limited for the Alpha device and phase-limited with the Beta device. C Average desired and measured torque from 100 steady-state walking steps.

exoskeleton, compliance in the shoe and heel string was sufficient to enable comfortable walking.

Additive manufacturing and lost-wax carbon fiber layups allowed for more organically shaped components, which reduced the overall envelope of the Beta exoskeleton. This is beneficial because large envelopes may require users to increase step width, resulting in higher metabolic energy consumption [19] and increased likelihood of collisions.

Anthropomorphic joints used in the Alpha and Beta designs made measurement of ankle angle simple and accurate. Double shear connections at medial and lateral joints in the Beta design resulted in consistent shaft and encoder alignment. Co-axial single shear joints used in the Alpha design were less robust to loading out of the sagittal plane. Removing the explicit joint from an exoskeleton can reduce overall device weight and envelope while not compromising structural strength [11]. However, This approach complicates measurement of the ankle angle, limiting the variety of control strategies that can be implemented.

While these exoskeletons are excellent research tools, they cannot be used autonomously. The high torque and bandwidth are primarily enabled by large off-board motors and controllers. Decoupling actuation from end-effector design, however, has allowed rapid design iteration.

Some designs require orthotists to fabricate custom interfaces for each user [24], which can improve fit, but at an additional monetary cost. The easily manufactured modular components of the Alpha and Beta exoskeletons allow for accurate alignment of the mechanical joint with the human joint for a wide range of users. However, making components in a variety of sizes and exchanging these components is time consuming. An adjustable design may reduce these costs, but often adds mass, increasing metabolic energy consumption during walking [15].

While leaf springs are theoretically much lighter than coil springs for a given stiffness, increased size and additional hardware for improved robustness can limit mass savings. The Alpha lever arm assembly, including the two leaf springs, aluminum cross-bar, and connective hardware, was only $19 \%$ lighter than the coil spring and titanium assembly of the Beta design. This comparison is confounded by the fact that the Beta exoskeleton was designed for larger loads than the Alpha design. The Beta exoskeleton originally used a fiberglass leaf spring, which made the assembly $0.040 \mathrm{~kg}$ lighter and lengthened the ankle lever arm, thereby reducing torques at the motor. This leaf spring proved unreliable, however, even after changes were made to the attachment hardware. The coil spring that replaced the leaf spring, though heavier, increased robustness and made interchanging springs of different stiffnesses easier.

Oscillations were present in the Bode plot phase diagram for the Alpha device at lower frequencies. These may be the result of un-modeled dynamics, particularly those of the tether and the human. Inspection of the time-series torque trajectory showed ripples at lower frequencies that may have been caused by changes on the human side of the system 
or oscillations in the Bowden cable transmission. Bandwidth tests could be improved by including more data in the lower frequency range. This could be achieved by commanding an exponential, rather than linear, chirp in desired torque for a longer duration.

Series elasticity plays a large role in torque tracking performance, but optimal spring stiffness may be a function of individual morphology, peak applied torques, and control strategies and might be difficult to predict. In pilot tests with the Beta device, we found that very stiff or very compliant elastic elements worsened torque tracking errors. This was not the case for a prosthetic device we developed [25], in which the Bowden cable itself provided sufficient series compliance. This may be because the prosthesis is in series with the limb, and therefore receives more predictable loading. We plan to perform experiments to characterize these relationships.

\section{CONCLusion}

We designed, manufactured, and tested two ankle-foot exoskeletons which proved to have high peak torque and bandwidth and low torque tracking errors. These devices allow accurate realization of a wide range of torque profiles, which will enable exploration of novel assistance strategies. Series elasticity, selective compliance, three-point attachment, formfitting components, double-shear joints, and powerful offboard motors facilitate effective interactions between the exoskeleton and the user.

The Alpha exoskeleton featured increased compliance in uncontrolled directions, inexpensive manufacturing, and lighter construction due to the use of leaf springs as both series elastic elements and lever arms. However, this design includes larger medial and posterior protrusions than the Beta device, which may result in less natural gait. The Beta device featured strain gauges which proved to be lighter, more accurate, and less expensive than the load cell used on the Alpha device. The coil spring, while heavier than leaf springs, is more robust and allows for simple adjustability of stiffness. While the flowing three-dimensional carbon fiber forms of the Beta device allowed for a close fit, they were time consuming to construct. These illustrate some of the trade-offs to consider in exoskeleton design.

The approaches demonstrated here could also be implemented in knee and hip exoskeletons, allowing researchers to explore biomechanical interactions across joints during locomotion as well as to analyze the effect of different assistance strategies.

\section{ACKNOWLEDGEMENTS}

The authors thank Jan Warnaars, Jessica Lee, Kristen Hauser, Justin Barsano, Sean Archie and Kyle Rawding for their help with design and fabrication of the devices and Padraig Taggart for help with figure and video editing.

\section{REFERENCES}

[1] D. Aoyagi, W. E. Ichinose, S. J. Harkema, D. J. Reinkensmeyer, and J. E. Bobrow, "A robot and control algorithm that can synchronously assist in naturalistic motion during body-weight-supported gait training following neurologic injury," Trans. Neural Syst. Rehabil. Eng., vol. 15 , no. 3 , pp. $387-400,2007$.

[2] E. Guizzo and T. Deyle, "Robotics trends for 2012," Rob. Autom. Mag., pp. 119-123, 2012.

[3] P. Malcolm, W. Derave, S. Galle, and D. De Clercq, "A simple exoskeleton that assists plantarflexion can reduce the metabolic cost of human walking," PLoS: ONE, vol. 8, no. 2, p. e56137, 2013.

[4] D. P. Ferris, K. E. Gordon, and G. S. Sawicki, "An improved powered ankle-foot orthosis using proportional myoelectric control," Gait Post., vol. 23, pp. 425-428, 2006.

[5] S. Au, M. Berniker, and H. Herr, "Powered ankle-foot prosthesis to assist level-ground and stair-descent gaits," Neur. Net., vol. 21, pp. 654-666, 2008.

[6] M. Kim and S. H. Collins, "Once-per-step control of ankle-foot prosthesis push-off work reduces effort associated with balance during walking," vol. in review, 2015.

[7] D. A. Winter, The Biomechanics and Motor Control of Human Gait: Normal, Elderly and Pathological. Waterloo, Canada: Waterloo Biomechanics, 1991.

[8] S. H. Collins and R. W. Jackson, "Inducing self-selected human engagement in robotic locomotion blah training," in Proc. Int. Conf. Rehab. Rob., 2013, pp. 1-6.

[9] J. Hidler, D. Nichols, M. Pelliccio, K. Brady, D. D. Campbell, J. H. Kahn, and T. G. Hornby, "Multicenter randomized clinical trial evaluating the effectiveness of the Lokomat in subacute stroke," Neurorehab. Neur. Rep., vol. 23, no. 1, pp. 5-13, 2009.

[10] G. S. Sawicki and D. P. Ferris, "Powered ankle exoskeletons reveal the metabolic cost of plantar flexor mechanical work during walking with longer steps at constant step frequency," The Journal of Experimental Biology, vol. 212, pp. 21-31, Jan. 2009.

[11] L. M. Mooney, E. J. Rouse, and H. M. Herr, "Autonomous exoskeleton reduces metabolic cost of human walking during load carriage," $J$. Neuroeng. Rehabil., vol. 11, p. 80, 2014.

[12] R. W. Jackson and S. H. Collins, "An experimental comparison of the relative benefits of work and torque assistance in ankle exoskeletons," J. Appl. Physiol., vol. in review, 2015.

[13] P. Malcolm, R. E. Quesada, J. M. Caputo, and S. H. Collins, "The influence of push-off timing in a robotic ankle-foot prostheses on the energetics and mechanics of walking," vol. 12 , no. 21 , p. in press, 2014.

[14] G. Pratt and M. Williamson, "Series elastic actuators," in Proc. Int. Conf. Intel. Rob. Sys., 1995.

[15] R. C. Browning, J. R. Modica, R. Kram, and A. Goswami, "The effects of adding mass to the legs on the energetics and biomechanics of walking," Med. Sci. Sports Exer., vol. 39, no. 3, pp. 515-525, 2007.

[16] D. P. Ferris, G. S. Sawicki, and M. A. Daley, "A physiologist's perspective on robotic exoskeletons for human locomotion," Int. J. Hum. Rob., vol. 4, no. 3, pp. 507-528, 2007.

[17] J. M. Donelan, Q. Li, V. Naing, J. A. Hoffer, D. J. Weber, and A. D. Kuo, "Biomechanical energy harvesting: Generating electricity during walking with minimal user effort," Science, vol. 319, no. 5864, pp. 807-810, 2008.

[18] J. M. Caputo and S. H. Collins, "A universal ankle-foot prosthesis emulator for experiments during human locomotion," J. Biomech. Eng., vol. 136, p. 035002, 2014.

[19] J. M. Donelan, R. Kram, and A. D. Kuo, "Mechanical and metabolic determinants of the preferred step width in human walking." Proc. Roy. Soc. Lon. B, vol. 268, pp. 1985-1992, 2001.

[20] M. P. Kadaba, H. K. Ramakrishnan, and M. E. Wootten, "Measurement of lower extremity kinematics during level walking," Journal of Orthopaedic Research, vol. 8, pp. 383-392, 1990.

[21] T. M. Owings and M. D. Grabiner, "Variability of step kinematics in young and older adults," pp. 24-29, 2004.

[22] J. Zhang, C. C. Cheah, and S. H. Collins, "Experimental comparison of torque control methods on an ankle exoskeleton during human walking," in Proc. Int. Conf. Rob. Autom., 2015.

[23] "Telecommunications: Glossary of telecommunication terms," Federal Standard 1037C, Aug. 1996.

[24] K. E. Gordon, D. P. Ferris, and A. D. Kuo, "Metabolic and mechanical energy costs of reducing vertical center of mass movement during gait," Arch. Phys. Med. Rehab., vol. 90, pp. 136-144, 2009.

[25] S. H. Collins, M. Kim, T. Chen, and T. Chen, "An ankle-foot prosthesis emulator with control of plantarflexion and inversion-eversion torque," in Proc. Int. Conf. Rob. Autom., 2015, p. accepted. 